\title{
Improving the Way We See: Adaptive Optics Based Optical Microscopy for Deep-Tissue Imaging
}

\author{
Pranoy Sahu ${ }^{1}$ and Nirmal Mazumder ${ }^{2 *}$ \\ ${ }^{1}$ Insitute of Biochemistry and Cell Biology, National Research Council, Naples, Italy, ${ }^{2}$ Department of Biophysics, Manipal \\ School of Life Sciences, Manipal Academy of Higher Education, Manipal, India
}

With the recent developments in optical imaging tools and techniques, scientists are now able to image deeper regions of the tissue with greater resolution and accuracy. However, light scattering while imaging deeper regions of a biological tissue remains a fundamental issue. Presence of lipids, proteins and nucleic acids in the tissue makes it inhomogeneous for a given wavelength of light. Two-photon fluorescence (TPF) microscopy supplemented with improved invasive optical tools allows functional imaging in awake behaving mammals in an unprecedented manner. Similarly, improved optical methods conjugated with previously existing scanning laser ophthalmoscopy (SLO) has paved diffraction-limited retinal imaging. With the evolving technology, scientists are now able to resolve biological structures and function at the sub-cellular level. Wavefront correcting methods like adaptive optics $(\mathrm{AO})$ has been implemented in correcting tissue or optical-based distortions, shaping the excitation beam in 3D-holography to target multiple neurons. And more recently, AO-based SLO is implemented for eye imaging both in research and clinical settings. In this review, we discuss some of the recent improvements in TPF microscopy with the application of AO for wavefront corrections and its recent application in brain imaging as well as ophthalmoscopy.

Keywords: adaptive optics, two-photon fluorescence, ophthalmoscopy, tissue imaging, brain

\section{INTRODUCTION}

In confocal microscopy the 3D resolution is mainly achieved from the light originating from focus and not from the scattered light, a common problem associated mainly while imaging deep tissues [1-3]. Light scattering could be reduced using longer excitation wavelengths [4-6], however, it is limited to certain wavelengths. Linear (one-photon) excitation microscopy is mainly suited for near-surface $(<100 \mu \mathrm{m})$ imaging. While non-linear (twophoton fluorescence) microscopy provides the advantage of imaging brain regions $>100 \mu \mathrm{m}$ with minimal scattering and better resolution. Deep tissue imaging at the single-cell level has provided adequate information on synaptic activity and function. Table 1 compares the with and without adaptive optics based confocal and two photon fluorescence microscopy. Conventional functional visualization neuroimaging techniques such as magnetic resonance imaging (MRI), positron emission tomography (PET), computerized tomography (CT) is currently used for imaging the human brain in vivo are usually expensive and have a poor spatial and temporal resolution [7]. The improvements in the optical approach have developed techniques that allow non-labeled imaging of tissues with high sensitivity, specificity, and spatial resolution under in vivo and in vitro conditions. Non-linear microscopy, in particular, TPF supplemented by the recent advances in optical labeling of deep tissues has become the method 
TABLE 1 | Comparison of microscopy techniques with and without Adaptive optics.

\begin{tabular}{|c|c|c|}
\hline & Confocal Microscopy & TPF-AO Microscopy \\
\hline Principle & $\begin{array}{l}\text { - Focuses excitation light to } \\
\text { the diffraction-limited spot. } \\
\text { - A detector uses a pinhole to } \\
\text { collect only focussed signal } \\
\text { photon and not } \\
\text { out-of-focus photons. }\end{array}$ & $\begin{array}{l}\text { - In AO based TPM, aberration } \\
\text { for the excitation light is } \\
\text { corrected (direct or indirect } \\
\text { AO) since imaging } \\
\text { performance depends on the } \\
\text { quality of excitation focus. }\end{array}$ \\
\hline $\begin{array}{l}\text { Imaging } \\
\text { depth }\end{array}$ & $\begin{array}{l}\text { - Applicable for thin } \\
\text { specimens or near surface } \\
\text { tissue regions }(<100 \mu \mathrm{m})\end{array}$ & $\begin{array}{l}\text { - Can be applicable for imaging } \\
\text { much deeper regions when } \\
\text { used with } \mathrm{Ti}^{\mathrm{A}} \mathrm{Al}_{2} \mathrm{O}_{3} \\
\text { regenerative amplifier (up } \\
\text { to } 1,000 \mu \mathrm{m} \text { ) }\end{array}$ \\
\hline $\begin{array}{l}\text { Sample } \\
\text { type }\end{array}$ & $\begin{array}{l}\text { - Mostly applicable for less } \\
\text { scattering samples like } \\
\text { monolayer cultures }\end{array}$ & $\begin{array}{l}\text { Due to use of less-scattering } \\
\text { long wavelength light, it is } \\
\text { widely applicable for imaging } \\
\text { deeper brain regions }\end{array}$ \\
\hline Advantages & $\begin{array}{l}\text { - Sensitivity of fluorescence } \\
\text { detection is very high in } \\
\text { presence of non-fluorescent } \\
\text { molecules } \\
\text { - It is best suited for gaining } \\
\text { cellular level information from } \\
\text { monolayer cultures }\end{array}$ & $\begin{array}{l}\text { - Captures even out-of-focus } \\
\text { photons emanating from the } \\
\text { specimen thereby increasing } \\
\text { fluorescence sensitivity } \\
\text { - Best suitable for deep tissue } \\
\text { imaging as the type of } \\
\text { aberration due to tissue } \\
\text { heterogeneity can be corrected } \\
\text { - Applicable for imaging } \\
\text { heterogeneously labeled } \\
\text { neuronal circuits }\end{array}$ \\
\hline Limitations & $\begin{array}{l}\text { - Only ballistic photons from } \\
\text { the specimen arrives the } \\
\text { detector. } \\
\text { - Tissue elucidation from } \\
\text { deeper regions (>100 } \mu \mathrm{m} \text { ) of } \\
\text { the brain is limited mainly by } \\
\text { the penetration of the } \\
\text { exciting laser caused by } \\
\text { scattering from } \\
\text { tissue heterogeneity }\end{array}$ & $\begin{array}{l}\text { - The increase in excitation } \\
\text { necessary to compensate } \\
\text { for signal loss compared } \\
\text { to fluorescence microscopy } \\
\text { further leads to photobleaching } \\
\text { and photodamage. } \\
\text { - Not applicable for studying } \\
\text { complex brain functions in live } \\
\text { behaving mammals }\end{array}$ \\
\hline
\end{tabular}

of choice for imaging intact tissues and live animals $[4,5,8]$. Principally, the TPF technique utilizes near-infrared wavelength and ultrashort pulses where the interaction of two photons usually of higher-order is responsible for contrast generation [9, 10]. Due to its high fluorescence intensity and depth resolution [2], TPF has drawn substantial attention for the study of deeper structures and vital functions of the brain and eye. Indeed, TPF coupled with fluorescence lifetime imaging (FLIM) has shown promising data on vitamin A metabolism in the eye to study the early onset of retinal diseases [11]. Owing to the heterogeneity of biological samples under investigation and the optical setup used, wavefront distortions limit the fluorescence signal-to-noise ratio and resolution with increasing depth [12]. AO based TPF overcomes such a loss in resolution by detecting and correcting the type of wavefront aberration generated while imaging scattering samples [13]. In the following sections, we will discuss some of the applications of AO in TPF based brain imaging.
Over the past decades, there have been great improvements in the field of ophthalmoscopy which has not only allowed scientists to successfully resolve fine retinal structures but also improved the clinical interventions for different eye diseases. This was mainly possible from the development of optical systems and recent advances in aberration correcting systems. However, when augmented with existing ophthalmoscopes such as conventional fundus imaging, SLO and spectral domainoptical coherence tomography (SD-OCT), AO enhances both structural and functional retinal imaging. First developed by David Williams and coworkers at the University of Rochester, $\mathrm{AO}$-fundus imaging was used to overcome wavefront aberrations in $<0.5 \mathrm{~s}$ and to obtain dynamic correction of fluctuations in Zernike mode [14]. SLO was first applied in the 1980s and is known to be the method of choice for clinical imaging with superior contrast and resolution. In SLO, retinal images are generated over time by measuring the scattered light as the focused beam scans across the retina surface. SLO provides enhanced image contrast mainly due to the "confocality" wherein light not originating from focus is rejected via a pinhole. Reflectance imaging is an application of SLO where the scattered light within an optically aberrated biological sample like an eye is measured. Such a technique, when used along with different wavelength lasers, could be used in clinical settings for multicolour imaging like fundus autofluorescence (FAF) [15] Although, being routinely used in clinical imaging of the eye, contrast and depth of imaging obtained in conventional SLO methods are limited. In the later part of the review, we highlight some of the recent advances in $\mathrm{AO}$ based retinal imaging.

Optical imaging is the foremost important method of choice for imaging biological specimens. With the recent developments in optical methods, three-dimensional and highly resolved structures from biological samples can be achieved. One of the limitations of deep brain imaging is the scattering of light through the heterogeneous tissues, which affects the penetration depth and image resolution. The advances in the TPF microscopy techniques provide a powerful tool for overcoming scattering of light, for in vivo neuro-imaging with sub-cellular resolution, endogenous contrast specificity, pinhole less optical sectioning capability, high penetration depth, and so on. The NIR light source is usually the choice for excitation source as it avoids water absorption, thus, reducing photo-bleaching and phototoxicity in the biological optical window, and also increases the penetration depth within the thick tissue $[2,3,16]$. Ability to image deeper brain regions with the single-cell resolution has refined the establishment of synaptic activity and its functions. The high fluorescence intensity and depth resolution assist in studying the structure and vital functions of the brain.

An optical system incorporates three major components, the laser system (pulsed), optical modalities (lenses, mirrors and objective etc.) and the specimen under investigation. Each of these components contributes toward the optical aberrations which in turn affects the imaging time and resolution. Although using aberration-corrected optical components reduce the imaging limitations when scanning deeper regions of a biological specimen, sample induced aberrations and scattering yet remains substantial. One such tool very quickly realized and 
implemented in optical imaging of biological samples is AO. For thick biological specimens, the optical signal strength reduces due to the aberration caused by the scattering medium (sample) and optical components used in the system $[17,18]$. The ability to correct the aberration and improve the signal strength has been demonstrated using adaptive optics integrated into a wavefront sensor (WS) or sensor-less AO [19]. In the case of sensor-based $\mathrm{AO}$, a WS estimates the aberration present in the imaging system and either a liquid crystal spatial light modulator (LC-SLM) or deformable membrane mirror membrane (DMM) corrects the aberration. Whereas, in sensor-less AO, the wavefront of the excitation beam is modulated and the optimal wavefront is determined through quantifying the properties of the acquired image in the form of intensity or sharpness in a feedback loop [18]. Sensorless corrections have particularly suffered from long optimization time and the introduction of fluorescent fiducial markers. In the following sections, we will briefly discuss the applications of AO in biological imaging using TPF and SLO.

\section{APPLICATION OF TPF-AO IN BIOLOGICAL IMAGING}

\section{AO in Brain Imaging}

Biological information from $3 \mathrm{D}$ structures such as the mammalian brain has consistently intrigued scientists over decades. However, it is quite challenging to achieve higherresolution images with optimum spatial and temporal resolution. Light from deeper regions of the brain often suffers aberration due to tissue heterogeneity or the optical setup. In recent years, scientists have successfully measured and overcome such aberrations using special optical elements, such as a deformable mirror (DM) or liquid crystal spatial light modulators (LC-SLM), along with Shack-Hartmann wavefront sensor (SH-WS) [20-22]. Wavefront distortions could be corrected directly by measuring the wavefront of the emanating signals using WS and SLM before allowing it to enter the detector [19,23]. Direct wavefront corrections serve well for in vitro cell models or transparent samples due to an ample amount of unscattered light reaching the wavefront sensor. More recently, a multi-actuator based adaptive lens $(\mathrm{AL})$ was implemented in specimens derived from wavefront corrections [24]. Comparing the image quality between SLM based multiphoton imaging and AL-based imaging, Bueno and colleagues demonstrated an alternative AO-based approach to improve two-photon deep-tissue imaging. DM and LC-SLM used for wavefront correction when combined with algorithms provide ample opportunities for scientists to obtain better images using TPF. AO based TPF is a versatile tool that not only improves the optical distortions but can improve the image quality from sparsely labeled samples at greater depths. Recently, Champelovier et al. demonstrated that $\mathrm{AO}$ can be applied to heterogeneously labeled cell populations such as CA1 neurons of the hippocampus to measure neuronal activity effectively. Using axial-lock modality authors were able to measure neuronal activity from hippocampal neurons that were conveniently filtered based on low or high fluorescence intensity [18]. Liu et al. investigated in vivo TPF imaging of fine dendritic structures in deep cortical layers of adult mice making use of a closed cranial window and a two-photon microscope. The full AO correction of TPF images of neurons with a yellow fluorescent protein (YFP) (Thy1-YFP SLICK A mice) was obtained at a depth of 690 and $770 \mu \mathrm{m}$ below the pia mater in the brain. This method efficiently corrects all aberrations including that of the microscope, cover glass, and brain tissue [25]. TPF serves as a valuable tool for the analysis of neuronal structures and functions, like long term potentiation, calcium signaling pathway, microglial movement, calcium dysregulation, synaptic plasticity, etc., which can all be used to study neurodegenerative disorders such as AD. TPM supplemented with improved invasive optical tools further allows imaging in awake behaving mammals in an unprecedented manner $[4,5]$. Use of high numerical aperture GRIN lens along with TPF-AO allows resolving minute synaptic structures from CA1 hippocampal neurons enabling recovery of diffraction-limited resolution. Moreover, AO based TPF enabled simultaneous calcium imaging from different hippocampal cells with a greater spatiotemporal resolution [26]. Two-photon $\mathrm{Ca}^{2+}$ imaging with the subcellular and temporal resolution is an important platform providing neuroscientists to visualize the cellular changes upon brain stimulation. To achieve greater resolution while imaging complex brain regions in brain slices or live mammals are challenging. Indeed, AO based TPF has been applied for $\mathrm{Ca}^{2+}$ imaging in live animals or fixed samples.

$\mathrm{AO}$ corrections have shown to improve in vivo $\mathrm{Ca}^{2+}$ imaging for monitoring neural activity [27]. $\mathrm{Ca}^{2+}$ imaging of neurons in the primary visual cortex of the mouse brain was performed without and with AO corrections (Figure 1) through a cranial window. An increase of 5-fold and 3-fold signal enhancement was observed while imaging fine structures and neuronal functionality, respectively. AO-based TPF benefits the studies of densely packed and labeled neuronal populations due to the ability to improve the signal-to-noise ratio and reduce the excitation volume at a depth. Being able to resolve brain structure and function not only by correcting wavefront distortions with AO but also shaping the excitation beam in 3D (holography) to target different neuronal populations in the intact brain is vital. Using TPF-AO based holography, Yang and Yuste developed a method wherein using SLMs to generate hologram to optically manipulate and image multiple neurons in 3D [21]. In TPF based brain imaging, sequential scanning of a laser beam across the entire sample slows down the imaging speed. This issue could be resolved by computer-generated holograms to image multiple cells at any given time. More importantly, AO can be now applied to achieve a lateral resolution which was not possible before to resolve individual rod and cone cells in the retina.

\section{AO-Based Retinal Imaging}

The human eye is an optical system where aberration is generated from the difference between a reference wavefront and an actual wavefront from every individual point on the pupil [28]. Since the human eye suffers from optical monochromatic aberrations, developing optical strategies to enhance the spatial resolution in retinal imaging became inevitable. Some of the early in vivo retinal imaging techniques were based on spectral and directional reflectance averaged 1-2 degrees of the retina which allowed 

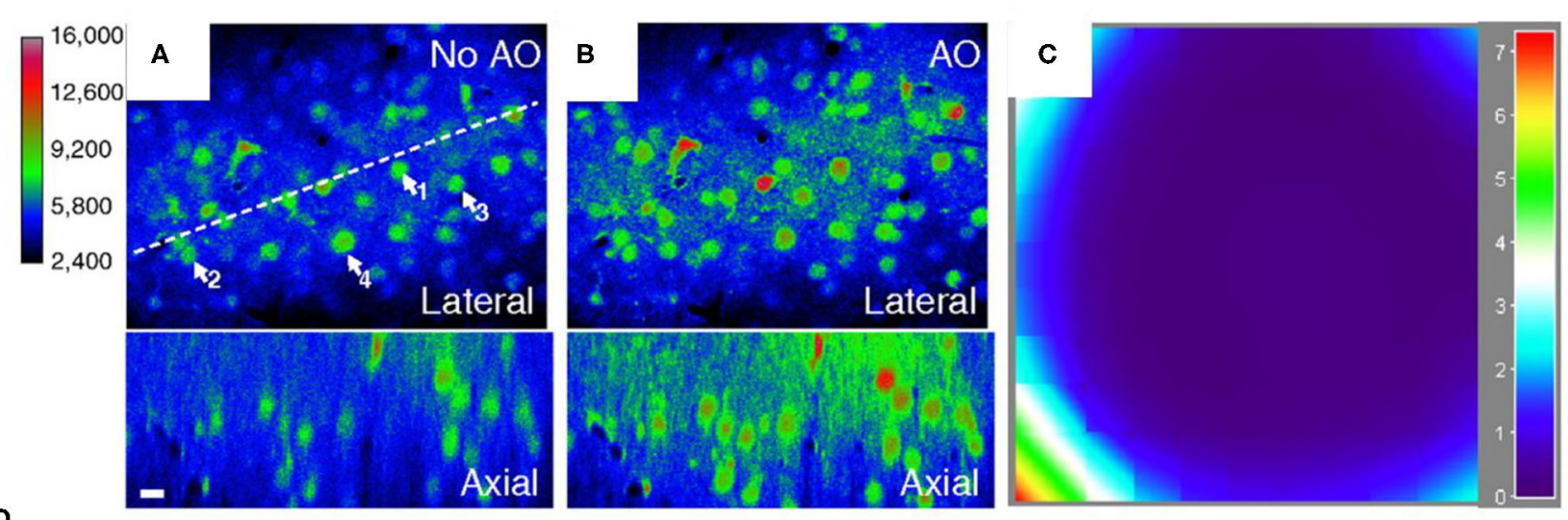

D

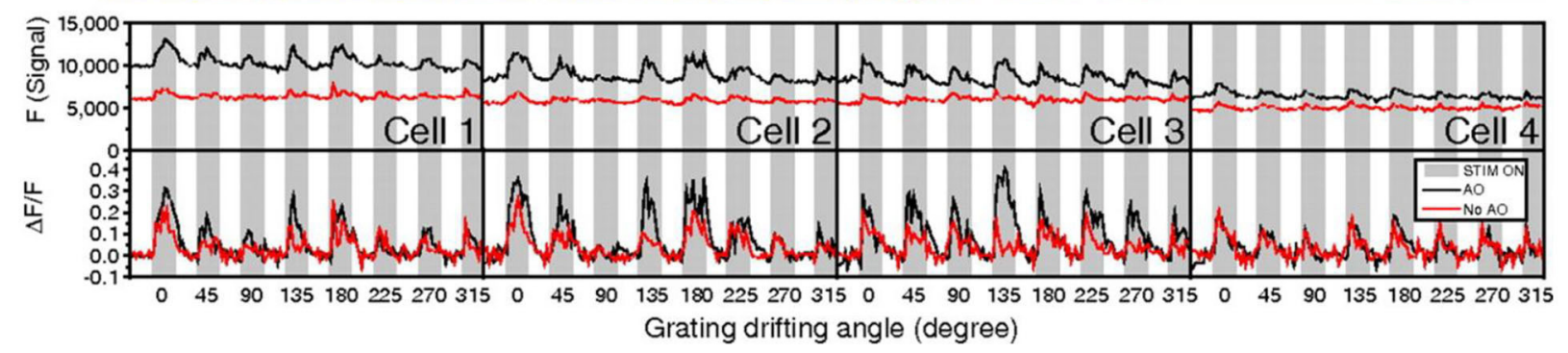

FIGURE 1 | Adaptive optics (AO) based image correction in vivo. (A) Two photon fluorescence lateral and axial images of OGB-1 AM labeled neurons at $155 \mu$ m below the brain surface in absence of $A O$ correction. (B) With AO correction. (C) Shows the measured aberration of excitation wavelength. (D) Fluorescence signal (black) and its percentage change (red) for four cells indicated in Figure (A) without and with AO correction. Time periods are denoted wit gray bars. Black-and-white gratings drift in eight different directions, from left to right, $0,45,90,135,180,225,270$, and $315^{\circ}$. This image is adapted with permission from [27].

studying structural and physiological aspects of the retina [29]. The first imaging modality developed to study retinal reflectance was the use of multi-wavelength video reflectometers [30] and scanning laser ophthalmoscopes developed by Robert Webb and coworkers [31]. Direct imaging of the retina at the cellular level (restricted only to sparsely distributed cones) was possible with a basic flood illuminated fundus camera. However, to achieve images of rods and cones with greater resolution from a broader population was only possible through AO based optical corrections. The first evidence of AO based retinal imaging came from a group in Heidelberg where SLO was previously developed [32]. Using deformable mirrors along with SLO, scientists were able to obtain much improved retinal images. Although the resolution generated by conventional SLO is hindered by the ocular aberrations, AO-SLO is the method of choice for overcoming ocular generated wavefront aberrations to enhance the imaging resolution of the mammalian eye (Figure 2). Conventional fundus imaging techniques such as fundus fluorescein angiography (FFA), optical coherence tomography (OCT) and fundus autofluorescence are insufficient in resolving fine structures like individual rods and cones or vessel and capillaries [34]. AO-SLO overcomes this limitation to resolve fine structures due to enhanced transverse resolution $(\sim 2.5 \mu \mathrm{m})$ while imaging the retina [35]. Studies have shown that while using a $50 \mu \mathrm{m}$ confocal pinhole axial resolution as low as $71 \mu \mathrm{m}$ could be achieved [36]. Moreover, the axial and lateral resolution in AO-SLO can be refined by adjusting the pinhole size of the system. Owing to its confocal nature, AO-SLO is widely used for visualizing different layers within the retina such as photoreceptors, nerve fibers, retinal pigment epithelium (RPE) [37].

Retinal ganglion cells are the primary cells that process visual information and conveys it to the brain. In clinical settings, loss or death of ganglion cells is the major cause of glaucoma. AOSLO provides a crucial imaging modality thus improving retinal ganglion cell imaging both for research and clinical purposes in a non-invasive manner [35]. Split-detector is a modality used in AO-SLO to image retinal structures such as rods and cones in the living eye of the patient and healthy control [38]. Alternatively, in vivo TPF used for functional retinal imaging has turned out to be an important tool to study individual retinal cells in primates when supplemented with an AO-based ophthalmoscope. It is now evident that TPF-AO-SLO could be implemented to detect fluorescence from retinal cells of a live animal with minimal photodamage to the fundus [39]. Thus, the AO based SLO approach is now widely applied for studying and monitoring age-related macular degeneration and vascular diseases with ease (reviewed elsewhere by [40]). Pinhas et al., applied AO-SLO in combination with both oral $(7 \mathrm{mg} / \mathrm{mL}$ ) and intravenous (500 mg) fluorescein to investigate microvasculature in humans at different retinal depths [41]. This study, one of many shows the clinical application of AO-SLO in improved lateral resolution to resolve fine retinal capillaries (average eye focal length of $\sim 17 \mathrm{~mm}$; pupil diameter of $7.75 \mathrm{~mm}$ and axial resolving limit of $20 \mu \mathrm{m}$ ). 


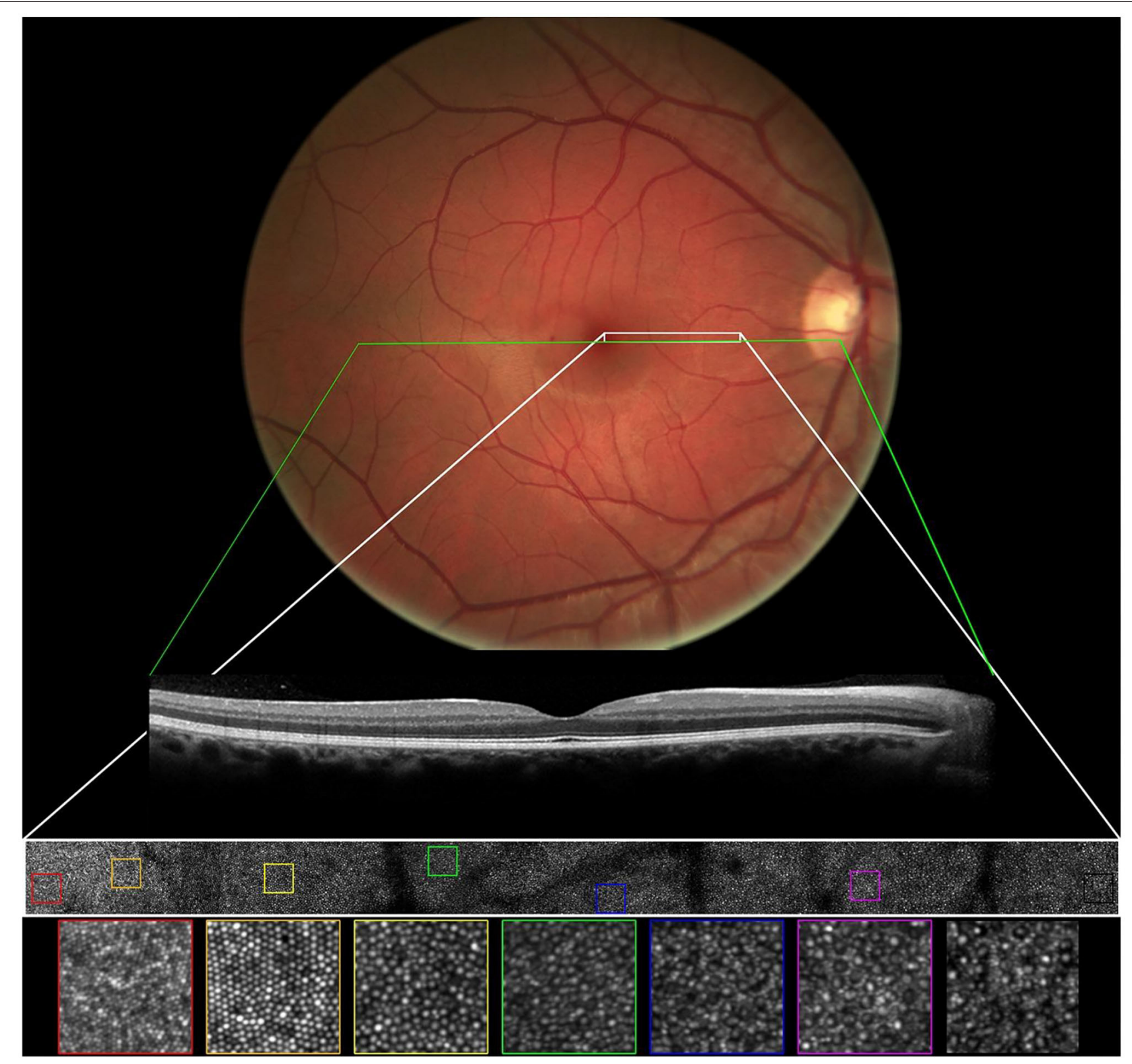

FIGURE 2 | Comparison of a conventional fundus photograph. SD-OCT cross sectional image obtained (green line) was overlaid on the fundus photograph and a montage of confocal AO-SLO images acquired (white box) was overlaid on the fundus image in the right eye of a 31 year old normal male. Magnified photoreceptor mosaic images from AO-SLO montage has been represented in colored boxes. This images has been adapted from [33] with permission.

AO-based OCT on the other hand is a label-free technique that improves lateral resolution $(\sim 4 \mu \mathrm{m})$ of the human eye while maintaining axial resolution $(\sim 6 \mu \mathrm{m})$ of the OCT alone. Huang and colleagues first showed the application of OCT in optical sectioning of biological samples such as the retina, which thereafter revolutionized the ophthalmoscopy field [42]. Importantly, in AO-OCT the axial and lateral resolution are decoupled, unlike SLO. The axial resolution of OCT is theoretically limited by the coherence properties of the imaging light source. As the human eye suffers from significant longitudinal aberration, i.e., different wavelengths focus on different planes, AO provides a way out to improve lateral resolution and sensitivity of OCT [43]. Such an improvement within the optical system has been widely used in 3D-imaging of photoreceptors and other cellular components within the retina $[44,45]$. Recently, a group from the School of Engineering Science in Canada implemented sensorless adaptive optics (SAO) guided by low-coherence gated OCT to improve two-photon excitation fluorescence image brightness and sharpness of mouse retina in vivo [46]. Using low-coherence guided optical plane 
selection, scientists were able to optimize the system in $60 \mathrm{~s}$ followed by TPF image acquisition at $100 \mathrm{~ms}$ per frame. A short optimisation protocol and improved image quality after aberration correction allowed imaging mouse retina in vivo in an unprecedented manner. Such AO based applications of optical imaging modalities are now paving their way into clinical settings to better understand the biological processes involved in disease generation and eventually improving existing therapeutic interventions.

\section{CONCLUSION AND FUTURE PERSPECTIVES}

The introduction of AO-based optical imaging has greatly improved deep-tissue imaging which was previously unattainable using conventional optical imaging microscopes. Since its usage in correcting atmospheric aberrations in telescopes, AO-based optical imaging has now paved its way into biological imaging. With AO, the diffraction-limited resolution could be easily achieved by correcting deep-tissue mediated aberrations. More importantly, the technology has improved the imaging quality and speed at which fine structural and functional aspects of living biological samples can be studied. The direct wavefront corrections (using $\mathrm{SH}$ wavefront sensors) provides a rapid and effective imaging modality compared to indirect wavefront sensing methods. Although the latter could be easily applied to any existing microscopes and is suitable for both scattering and transparent samples. Imaging deeper regions within a biological tissue have mostly been overcome by the implementation of AO based corrections. Although imaging complex neuronal populations with an AO-based optical system needs further improvements, there has been considerable improvement in retinal imaging irrespective of the differences arising from refractive index or age. Yet, imaging deeper neuronal populations with enhanced spatial-temporal resolution in a freely moving animal remain challenging. While recording neural activity in awake animals, brain movement remains a serious concern and are corrected by post image processing of 2D images. However, this approach is slow and cannot correct axial movements in a small region of interest and photostimulation. In 2016 Nadella and coworkers developed a 3D random-access twophoton laser scanning microscope using a rapid closed-loop system and acousto-optic lens (AOL). This system improves motion-mediated artifacts and monitors real-time 3D brain tissue movement at up to $1 \mathrm{kHz}$ with submicrometer spatial resolution in behaving mice and zebrafish [47]. In another study, a compact AOL two-photon microscope was used for highspeed line scanning within an imaging volume at $35-50 \mathrm{kHz}$ up to hundreds of micrometers. It was able to monitor cerebellar interneurons sparsely distributed neuronal activity over large depth ranges in awake animals [48].

Miniaturized fluorescence microscopes or miniscopes are becoming a key tool in neuroscience research owing to their high-resolution deep brain imaging in freely behaving animals.
Owing to their lightweight, miniscopes utilizing one-photon or two-photon excitation modalities have been reported in the study of cellular activity when coupled to a freely moving animal [49, 50]. Miniscopes provides convenient in vivo deep-tissue imaging in an awake free moving animal due to, $i$ ) it's decreased footprint and weight ii) easy mounting on small animals iii) ability to record multiple neuronal activities simultaneously, thereby aiding in better understanding inter-regional signaling in behaving animal models [51].

Two-photon microscopy serves as a method of choice over conventional microscopy techniques for deep-tissue imaging. However, due to the scattering of ballistic photons from deeper samples, an optical method that could limit photon loss is necessary. TPF augmented with AO provides the advantage of limiting photon loss from deep biological samples such as the human brain [3] and eye [35]. AO-TPF has been incorporated in neurobiology and ophthalmology labs across the globe to achieve diffraction-limited contrast in biological imaging $[14,52,53]$. The use of AO-based optical imaging in ophthalmology has greatly changed the way we see the human eye both at the cellular and metabolic level. Integrating sensorless $\mathrm{AO}$ along with dispersion compensation of ultrashort laser pulse can improve TPF intensity of endogenous retinal fluorophores. Indeed, the use of ultrashort laser (20-fs pulse) did not show any modifications in function as well as the structure of the retina in vivo. Interestingly, the broad bandwidth of short pulses excites multiple fluorophores which provides better applications while studying cellular metabolism [11]. AO-based ophthalmoscopes also provide a platform to study several genetic diseases of the eye. AO-SLO has not only improved eye imaging but also facilitated its application in clinics for studying and monitoring retina-related conditions such as macular degeneration and Stargardt disease [54]. Above all, with the current advances in the filed of AO and TPF scientists can effortlessly image different regions within the brain or eye of a live organism which was impossible previously. With further improvements in the existing fluorescent probes, AO-based biological imaging modalities would further improve our way of seeing things in vivo.

\section{AUTHOR CONTRIBUTIONS}

PS wrote the manuscript. NM structure the manuscript and wrote the manuscript. Both authors contributed to the article and approved the submitted version.

\section{FUNDING}

NM thank the Department of Science and Technology (DST), Government of India (Project Number - DST/INT/BLG/P03/2019) for financial support. We thank Manipal School of Life Sciences (MSLS), Manipal Academy of Higher Education (MAHE), Manipal, Karnataka, India, for providing the infrastructure needed. PS thank Euro Bioimaging for the financial support. 


\section{REFERENCES}

1. Lu L, Gutruf P, Xia L, Bhatti DL, Wang X, Vazquez-Guardado A, et al. Wireless optoelectronic photometers for monitoring neuronal dynamics in the deep brain. Proc Natl Acad Sci. (2018) 115:E1374-83. doi: 10.1073/pnas.1718721115

2. Matsumoto N, Inoue T, Matsumoto A, Okazaki S. Correction of depthinduced spherical aberration for deep observation using two-photon excitation fluorescence microscopy with spatial light modulator. Biomed Opt Expr. (2015) 6:2575-87. doi: 10.1364/BOE.6.002575

3. Helmchen F, Denk W. Deep tissue two-photon microscopy. Nat Methods. (2005) 2:932-40. doi: 10.1038/nmeth818

4. Barretto RP, Ko TH, Jung JC, Wang TJ, Capps G, Waters AC, et al. Timelapse imaging of disease progression in deep brain areas using fluorescence microendoscopy. Nat Med. (2011) 17:223-8. doi: 10.1038/nm.2292

5. Resendez SL, Stuber GD. In vivo calcium imaging to illuminate neurocircuit activity dynamics underlying naturalistic behavior. Neuropsychopharmacology. (2015) 40:238. doi: 10.1038/npp.2014.206

6. Nimmerjahn A, Kirchhoff F, Helmchen F. Resting microglial cells are highly dynamic surveillants of brain parenchyma in vivo. Science. (2005) 308:1314-8. doi: 10.1126/science.1110647

7. Ross CA, Poirier MA. Protein aggregation and neurodegenerative disease. Nat Med. (2004) 10:S10-7. doi: 10.1038/nm1066

8. Mertz J, Nonlinear microscopy: new techniques and applications. Curr Opin Neurobiol. (2004) 14:610-6. doi: 10.1016/j.conb.2004.08.013

9. Periasamy A, Mazumder N, Sun Y, Christopher KG, Day RN. FRET Microscopy: Basics, Issues and Advantages of FLIM-FRET Imaging, Advanced Time-Correlated Single Photon Counting Applications. Cham: Springer (2015). p. 249-76. doi: 10.1007/978-3-319-14929-5_7

10. Mazumder N, Lyn RK, Singaravelu R, Ridsdale A, Moffatt DJ, Hu C-W, et al. Fluorescence lifetime imaging of alterations to cellular metabolism by domain 2 of the hepatitis C virus core protein. PLoS ONE. (2013) 8:e66738. doi: 10.1371/journal.pone.0066738

11. Palczewska G, Stremplewski P, Suh S, Alexander N, Salom D, Dong Z, et al. Two-photon imaging of the mammalian retina with ultrafast pulsing laser. JCI Insight. (2018) 3:e121555. doi: 10.1172/jci.insight.121555

12. Sahu P, Mazumder N. Advances in adaptive optics-based two-photon fluorescence microscopy for brain imaging. Lasers Med Sci. (2020) 35:317-28. doi: 10.1007/s10103-019-02908-z

13. Débarre D, Botcherby EJ, Watanabe T, Srinivas S, Booth MJ, Wilson T. Imagebased adaptive optics for two-photon microscopy. Opt Lett. (2009) 34:2495-7. doi: 10.1364/OL.34.002495

14. Hofer H, Chen L, Yoon G-Y, Singer B, Yamauchi Y, Williams DR. Improvement in retinal image quality with dynamic correction of the eye's aberrations. Opt Express. (2001) 8:631-43. doi: 10.1364/OE.8.000631

15. Calvo-Maroto AM, Esteve-Taboada JJ, Pérez-Cambrodí RJ, Madrid-Costa D, Cerviño A. Pilot study on visual function and fundus autofluorescence assessment in diabetic patients. J Ophthalmol. (2016) 2016:1287847. doi: $10.1155 / 2016 / 1287847$

16. Lu J, Li C, Singh-Alvarado J, Zhou ZC, Fröhlich F, Mooney R, et al. MIN1PIPE: a miniscope 1-photon-based calcium imaging signal extraction pipeline. Cell Rep. (2018) 23:3673-84. doi: 10.1016/j.celrep.2018.05.062

17. Yang W, Miller J-EK, Carrillo-Reid L, Pnevmatikakis E, Paninski L, Yuste R, et al. Simultaneous multi-plane imaging of neural circuits. Neuron. (2016) 89:269-84. doi: 10.1016/j.neuron.2015.12.012

18. Champelovier D, Teixeira J, Conan J-M, Balla N, Mugnier L, Tressard T, et al. Image-based adaptive optics for in vivo imaging in the hippocampus. Sci Rep. (2017) 7:42924. doi: 10.1038/srep42924

19. Galwaduge P, Kim S, Grosberg L, Hillman E. Simple wavefront correction framework for two-photon microscopy of in-vivo brain. Biomed Opt Expr. (2015) 6:2997-3013. doi: 10.1364/BOE.6.002997

20. Dal Maschio M, Donovan JC, Helmbrecht TO, Baier H. Linking neurons to network function and behavior by wo-photon holographic optogenetics and volumetric imaging. Neuron. (2017) 94:774-89.e5. doi: 10.1016/j.neuron.2017.04.034

21. Yang W, Yuste R. Holographic imaging and photostimulation of neural activity. Curr Opin Neurobiol. (2018) 50:211-21. doi: 10.1016/j.conb.2018.03.006
22. Yang W, Carrillo-Reid L, Bando Y, Peterka DS, Yuste R. Simultaneous twophoton imaging and two-photon optogenetics of cortical circuits in three dimensions. Elife. (2018) 7:e32671. doi: 10.7554/eLife.32671

23. Facomprez A, Beaurepaire E, Débarre D. Accuracy of correction in modal sensorless adaptive optics. Opt Express. (2012) 20:2598-612. doi: 10.1364/OE.20.002598

24. Bueno JM, Skorsetz M, Bonora S, Artal P. Wavefront correction in twophoton microscopy with a multi-actuator adaptive lens. Opt Express. (2018) 26:14278-87. doi: 10.1364/OE.26.014278

25. Liu R, Li Z, Marvin JS, Kleinfeld D. Direct wavefront sensing enables functional imaging of infragranular axons and spines. Nat Methods. (2019) 16:615-8. doi: 10.1038/s41592-019-0434-7

26. Qin Z, He S, Yang C, Yung JS-Y, Chen C, Leung CK-S, et al. Adaptive optics two-photon microscopy enables near-diffraction-limited and functional retinal imaging in vivo. Light Sci Appl. (2020) 9:1-11. doi: 10.1038/s41377-020-0317-9

27. Ji N, Sato TR, Betzig E. Characterization and adaptive optical correction of aberrations during in vivo imaging in the mouse cortex. Proc Natl Acad Sci. (2012) 109:22-7. doi: 10.1073/pnas.1109202108

28. Venkateswaran N, Galor A, Wang J, Karp CL. Optical coherence tomography for ocular surface and corneal diseases: a review. Eye Vision. (2018) 5:1-11. doi: 10.1186/s40662-018-0107-0

29. Delori FC, Noninvasive technique for oximetry of blood in retinal vessels. Appl Opt. (1988) 27:1113-25. doi: 10.1364/AO.27.001113

30. Faulkner D, Kemp C. Human rhodopsin measurement using a TVbased imaging fundus reflectometer. Vision Res. (1984) 24:221-31. doi: 10.1016/0042-6989(84)90124-X

31. Webb RH, Hughes GW, Pomerantzeff O. Flying spot TV ophthalmoscope. Appl Opt. (1980) 19:2991-7. doi: 10.1364/AO.19.002991

32. Dreher AW, Bille JF, Weinreb RN. Active optical depth resolution improvement of the laser tomographic scanner. Appl Opt. (1989) 28:804-8. doi: 10.1364/AO.28.000804

33. Morgan JI, The fundus photo has met its match: optical coherence tomography and adaptive optics ophthalmoscopy are here to stay. Ophthalmic Physiol Opt. (2016) 36:218-39. doi: 10.1111/opo.12289

34. Zhang B, Li N, Kang J, He Y, Chen X-M. Adaptive optics scanning laser ophthalmoscopy in fundus imaging, a review and update. Int J Ophthalmol. (2017) 10:1751. doi: 10.18240/ijo.2017.11.18

35. Rossi EA, Granger CE, Sharma R, Yang Q, Saito K, Schwarz C, et al. Imaging individual neurons in the retinal ganglion cell layer of the living eye. Proc Natl Acad Sci. (2017) 114:586-91. doi: 10.1073/pnas.1613445114

36. Romero-Borja F, Venkateswaran K, Roorda A, Hebert T. Optical slicing of human retinal tissue in vivo with the adaptive optics scanning laser ophthalmoscope. Appl Opt. (2005) 44:4032-40. doi: 10.1364/AO.44.0 04032

37. Godara P, Dubis AM, Roorda A, Duncan JL, Carroll J. Adaptive optics retinal imaging: emerging clinical applications. Optom Vis Sci. (2010) 87:930. doi: 10.1097/OPX.0b013e3181ff9a8b

38. Sun LW, Johnson RD, Langlo CS, Cooper RF, Razeen MM, Russillo $\mathrm{MC}$, et al. Assessing photoreceptor structure in retinitis pigmentosa and Usher syndrome. Invest Ophthalmol Vis Sci. (2016) 57:2428-42. doi: $10.1167 /$ iovs.15-18246

39. Hunter JJ, Masella B, Dubra A, Sharma R, Yin L, Merigan WH, et al. Images of photoreceptors in living primate eyes using adaptive optics two-photon ophthalmoscopy. Biomed Opt Expr. (2011) 2:139-48. doi: 10.1364/BOE.2.000139

40. Paques M, Meimon S, Rossant F, Rosenbaum D, Mrejen S, Sennlaub F, et al. Adaptive optics ophthalmoscopy: application to age-related macular degeneration and vascular diseases. Prog Retin Eye Res. (2018) 66:1-16. doi: 10.1016/j.preteyeres.2018.07.001

41. Pinhas A, Dubow M, Shah N, Chui TY, Scoles D, Sulai YN, et al. In vivo imaging of human retinal microvasculature using adaptive optics scanning light ophthalmoscope fluorescein angiography. Biomed Opt Expr. (2013) 4:1305-17. doi: 10.1364/BOE.4.001305

42. Huang D, Swanson EA, Lin CP, Schuman JS, Stinson WG, Chang W, et al. Optical coherence tomography. Science. (1991) 254:1178-81. doi: $10.1126 /$ science. 1957169 
43. Hermann B, Fernández E, Unterhuber A, Sattmann H, Fercher A, Drexler W, et al. Adaptive-optics ultrahigh-resolution optical coherence tomography. Opt Lett. (2004) 29:2142-4. doi: 10.1364/OL.29.002142

44. Zawadzki RJ, Cense B, Zhang Y, Choi SS, Miller DT, Werner JS. Ultrahigh-resolution optical coherence tomography with monochromatic and chromatic aberration correction. Opt Express. (2008) 16:8126-43. doi: 10.1364/OE.16.008126

45. Torti C, PovaŽay B, Hofer B, Unterhuber A, Carroll J, Ahnelt PK, et al. Adaptive optics optical coherence tomography at 120,000 depth scans/s for non-invasive cellular phenotyping of the living human retina. Opt Express. (2009) 17:19382-400. doi: 10.1364/OE.17.019382

46. Cua M, Wahl DJ, Zhao Y, Lee S, Bonora S, Zawadzki RJ, et al. Coherence-gated sensorless adaptive optics multiphoton retinal imaging. Sci Rep. (2016) 6:1-9. doi: 10.1038/srep32223

47. Nadella KNS, Roš H, Baragli C, Griffiths VA, Konstantinou G, Koimtzis T, et al. Random-access scanning microscopy for 3D imaging in awake behaving animals. Nat Methods. (2016) 13:1001-4. doi: 10.1038/nmeth.4033

48. Griffiths VA, Valera AM, Lau JY, Roš H, Younts TJ, Marin B, et al. Real-time 3D movement correction for two-photon imaging in behaving animals. Nat Methods. (2020) 17:741-8. doi: 10.1038/s41592-020-0851-7

49. Helmchen F, Fee MS, Tank DW, Denk W. A miniature head-mounted twophoton microscope: high-resolution brain imaging in freely moving animals. Neuron. (2001) 31:903-12. doi: 10.1016/S0896-6273(01)00421-4

50. Heo CH, Sarkar AR, Baik SH, Jung TS, Kim JJ, Kang H, et al. A quadrupolar two-photon fluorescent probe for in vivo imaging of amyloid- $\beta$ plaques. Chem Sci. (2016) 7:4600-6. doi: 10.1039/C6SC00355A
51. de Groot A, van den Boom BJ, van Genderen RM, Coppens J, van Veldhuijzen J, Bos J, et al. NINscope, a versatile miniscope for multiregion circuit investigations. Elife. (2020) 9:e49987. doi: 10.7554/eLife. 49987

52. Wang C, Liu R, Milkie DE, Sun W, Tan Z, Kerlin A, et al. Multiplexed aberration measurement for deep tissue imaging in vivo. Nat Methods. (2014) 11:1037-40. doi: 10.1038/nmeth.3068

53. Ruminski D, Palczewska G, Nowakowski M, Zielińska A, Kefalov VJ, Komar K, et al. Two-photon microperimetry: sensitivity of human photoreceptors to infrared light. Biomed Opt Expr. (2019) 10:4551-67. doi: 10.1364/BOE.10.004551

54. Song H, Rossi EA, Latchney L, Bessette A, Stone E, Hunter JJ, et al. Cone and rod loss in Stargardt disease revealed by adaptive optics scanning light ophthalmoscopy. JAMA Ophthalmol. (2015) 133:1198-203. doi: 10.1001/jamaophthalmol.2015.2443

Conflict of Interest: The authors declare that the research was conducted in the absence of any commercial or financial relationships that could be construed as a potential conflict of interest.

Copyright $\odot 2021$ Sahu and Mazumder. This is an open-access article distributed under the terms of the Creative Commons Attribution License (CC BY). The use, distribution or reproduction in other forums is permitted, provided the original author(s) and the copyright owner(s) are credited and that the original publication in this journal is cited, in accordance with accepted academic practice. No use, distribution or reproduction is permitted which does not comply with these terms. 